\title{
Study of Turbulent Flow using Half-Fourier Echo-Planar Imaging
}

\author{
A. O. Rodríguez \\ Centro de Investigación en Imagenologia e Instrumentación Médica, Universidad Autónoma Metropolitana Iztapalapa, \\ Av. San Rafael Atlixco 186, México, D. F., 09340. México
}

Received on 16 May, 2005; accepted on 28 November, 2005

\begin{abstract}
The Echo-Planar Imaging technique combined with a partial Fourier acquisition method was used to obtain velocity images for liquid flows in a circular cross-section pipe at Reynolds number of up to 8000 . This partial-Fourier imaging scheme is able to generate shorter echo times than the full-Fourier Echo-Planar Imaging methods, reducing the signal attenuation due to $\mathrm{T} 2 *$ and flow. Velocity images along the $\mathrm{z}$ axis were acquired with a time-scale of $80 \mathrm{~ms}$ thus obtaining a real-time description of flow in both the laminar and turbulent regimes. Velocity values and velocity fluctuations were computed with the flow image data. A comparison plot of NMR velocity and bulk velocity and a plot of velocity fluctuations were calculated to investigate the feasibility of this imaging technique. Flow encoded Echo-Planar Imaging together with a reduced data acquisition method can provide us with a real-time technique to capture instantaneous images of the flow field for both laminar and turbulent regimes.
\end{abstract}

Keywords: Turbulent flow; Imaging; Echo-planar imaging

\section{INTRODUCTION}

In order to distinguish transient phenomena with NMR imaging, the use of ultra-fast flow imaging techniques is required. Under these circumstances it is essential to have an observation time short relative to the time scale of the physical property to be measured. Transient phenomena include, for example, turbulent flow at high Reynolds number, flow and dispersion through porous media, and extensional deformation. It has been proved that Echo-Planar Imaging can successfully quantified spatial and temporal characteristics of turbulent flow in pipes [1-5]. Other flow MRI techniques have been proposed by other groups [6-8].

In this paper, a variant of the Echo-Planar Imaging (EPI) technique [1] is used to determine flow for the laminar and turbulent regimes. This version includes a partial Fourier technique [9] which is able to generate shorter echo times than the full-Fourier methods such Modulos Blipped Echo-Planar Imaging Single Shot (MBEST) [10], reducing the signal attenuation due to $\mathrm{T}^{*}$ and flow. The real-time flow measurement method proposed by Guilfoyle and et al. [1] was used to measure the velocity in the laminar regime and turbulent regimen. This mathematical expression was also used to derive a formula for the turbulent case. Then, a combination of the method above and a scheme introduced by Gatenby and Gore was used to measure the velocity fluctuations of the turbulent regime [1].

Gatenby and Gore have developed a phase-dependent expression to measure the turbulence intensity as a function of the gradient waveform and velocity fluctuation. We used the theoretical model of [1] for the long-lived regime together with a modified version of the flow EPI technique, and called Half Fourier Echo-Planar Imaging (HF EPI) [9], to measure turbulence intensity in pipe flows. Flow images for both regimes were acquired and their corresponding velocity values were also computed to investigate the feasibility of this MRI technique. In this paper, we present the results of studies of laminar and turbulent flow using Half-Fourier EPI. Our specific aim was to investigate whether our flow imaging technique was able to measure both quantitative and qualitative information in real time for the laminar flow and turbulent flow.

\section{METHOD}

A flow-encoding gradient is used prior to the EPI experiment incorporating a $180^{\circ}$ pulse to overcome magnetic field inhomogeneities. The flow-encoding sequence $90^{\circ}-\tau-180^{\circ}-$ $90_{x o r y}^{0}$ or $y$ pulse train prior to the standard EPI sequence. This is depicted in Fig. 1. Each gradient is applied for a time t. After the second flow encoding gradient pulse, the spin magnetisation is tipped back along the main field axis with a $90^{\circ}$ pulse. A spin phase scrambling gradient is then applied to destroy any remaining signal before applying a normal EPI experiment to image the residual longitudinal magnetisation created by the preceding $90^{\circ}$ pulse of the flow-encoding sequence. Two components of the magnetisation are considered; the first from the static spins $M_{\text {stat }}\left(=M_{x}=M_{0} \cos (\beta)\right)$ and other from the spins $M_{V}\left(=M_{y}=M_{0} \sin (\beta)\right)$, flowing with constant velocity $V$, which undergone a phase shift $\beta$ given by [1]:

$$
\beta=\gamma G V_{N M R}\left(\tau^{2}+\tau t_{r}\right)
$$

where $\gamma$ is gyromagnetic ratio, $t$ and $G$ are the duration time and strength of the gradient, $\tau$ is the delay time. The phase variation is directly proportional to the spin velocity.

The flow experiment was performed by supplying water through a circular pipe of $2.5 \mathrm{~cm}$ internal diameter from a water tap. A dual coil systems was used consisting of a whole body coil for excitation and a circular-shaped coil for reception. A $2 \mathrm{~m}$ inlet was provided to ensure that flow was fully developed by the time it reached the imaging coil. The slice thickness and in-plane resolution was $1 \mathrm{~cm}$ and $2.5 \mathrm{~mm}$. Transaxial 128x128 images were generated from Half Fourier sequence in 72 echoes were acquired in $70 \mathrm{~ms}$. The central 16 


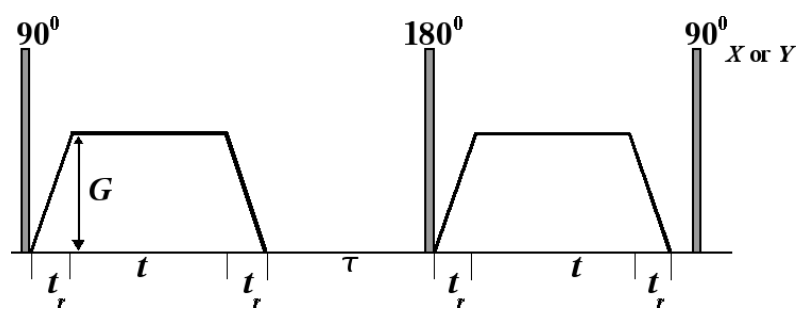

FIG. 1: Timing diagram for the RF pulses and flow-encoding gradients.

echoes were used to generate a coarse map, which was used subsequently in phase correction [9]. Images for each component were acquired for diferentes flow rates whit the following acquisition parameters: $t=12 \mathrm{~ms}$ and $G=4 \mathrm{mT} / \mathrm{cm}$, $t_{r}=400 \mu s$ (rise time), and $\tau=2 \mathrm{~ms}$ in Fig. 1. Each experiment was repeated for flow rates from 0 to $1770 \mathrm{ml} / \mathrm{cm}(0-30 \mathrm{~cm} / \mathrm{s})$. This range allows both laminar flow and turbulent flow to be developed. All experiments were performed on the Nottingham 0.5T imager.

The flow encoding sequence which proceeded the HF EPI read-out module is shown in Fig. 1. The magnitude of the signal is:

$$
\begin{aligned}
& S_{A}=S_{0} \sin (\beta) \\
& S_{B}=S_{0} \cos (\beta)
\end{aligned}
$$

depending upon whether the 2nd RF pulse is applied along the $X$ (cosine) or $Y$ (sine) axis in the rotating frame [1,9]. $\beta$ is the phase shift of the spins flowing along the pipe. We computed the velocity for the two regions, laminar and turbulent using two different expressions. To measure the flow in the laminar region the following velocity expression was derived from Eq. (1):

$$
V_{N M R}=\frac{\arctan \left(S_{A} / S_{B}\right)}{\gamma G\left(\tau^{2}+\tau t_{r}\right)}
$$

From the signal expression from [11] for the case of longlived fluctuations compared to both $t$ and $\tau$, and the flowsensitive gradient diagram in Fig. 1, the velocity fluctuation is:

$$
\left\langle u^{2}\right\rangle=\frac{\arctan \left(S_{A} / S_{B}\right)}{\gamma^{2} G^{2}\left(t^{2}+\tau\left(t+t_{r}\right)+3 t t_{r}+2 t_{r}^{2}\right)^{2}}
$$

where $t_{r}$ is the rise time, the variable $\beta$ can be the velocity $V_{N M R}$ (laminar flow) of Eq. (1) or the velocity fluctuation $\left\langle u^{2}\right\rangle$ (turbulent flow) of Eq. (4).

\section{RESULTS AND DISCUSSION}

Half-Fourier EP images were acquire of a circular cross section for the static case, laminar and turbulent regime. Flow

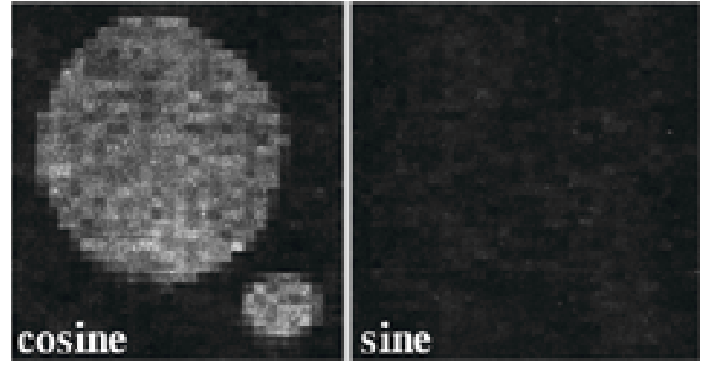

FIG. 2: Phantom images of stationary water: sine (weak) and cosine (strong) components.

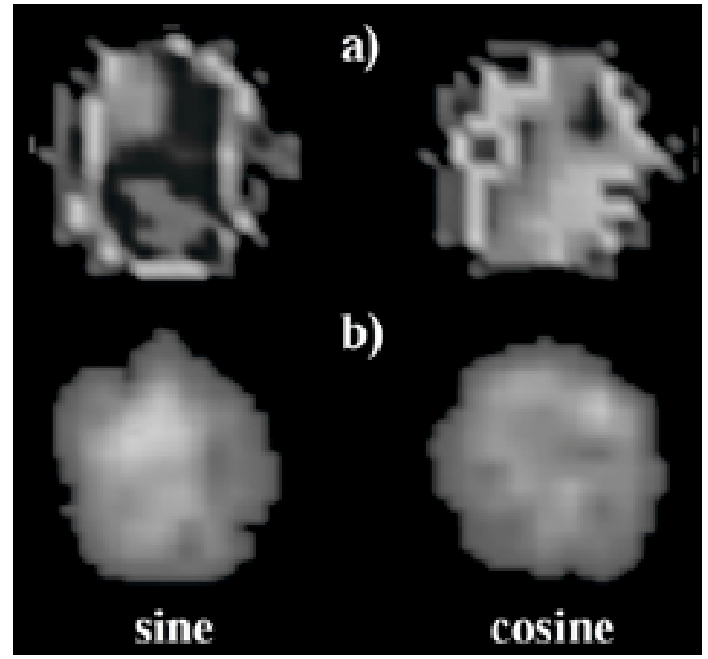

FIG. 3: Phantom images of components sine and cosine for: a) laminar regime and b) turbulent regime.

images for each flow rate were obtained and their corresponding velocities were computed with the pipe geometry and the flow rate. Fig. 2 shows two cross-sectional images of the water phantom (right lower corner) and pipe for the static case. Sine and cosine components for the laminar and turbulence cases were also computed and shown in Fig. 3. From the images showing laminar behaviour a velocity profile was plotted and shown in Fig. 4. Fig. 5 shows a graph of the NMR velocity calculated from the flow images and Eq. (3) against the Bulk velocity computed as mentioned above. The linear regression of the experimental data stresses the excellent agreement of the NMR velocity the actual bulk velocity in the pipe. Thus, it can be implied that NMR velocity $=$ Bulk velocity. This indicates a linear function over the laminar range as expected for the laminar behaviour. Fig. 6a) shows that the experimentallyacquired values of $\left\langle u^{2}\right\rangle$ plotted against Reynold's number. There is no need to use a general fit to calculate the velocity fluctuations for the long-lived regime as reported in [11].

Gatenby and Gore reported a constant value of $\left\langle u^{2}\right\rangle$ using a gradient-recalled echo sequence for an $\operatorname{Re}<2000$. The velocity fluctuation measured with HF EPI has almost no variation for an $\operatorname{Re}<100$, and contrary to results previously published a linear dependence can be appreciated between 100 and 3000 . Despite flow behaves in a laminar fashion a proportional in- 


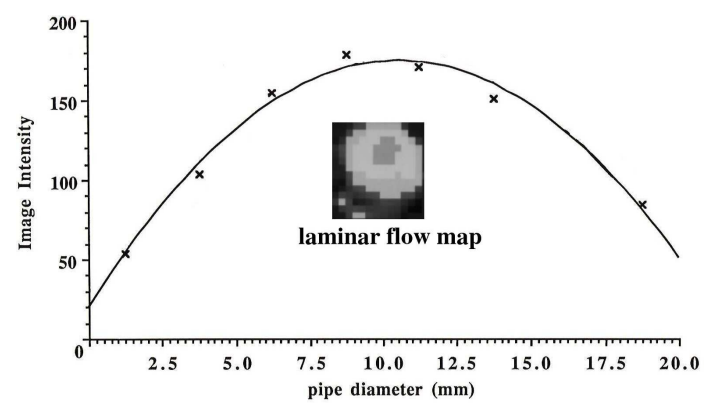

FIG. 4: Velocity profile of laminar flow obtained in transverse section across the circular pipe.

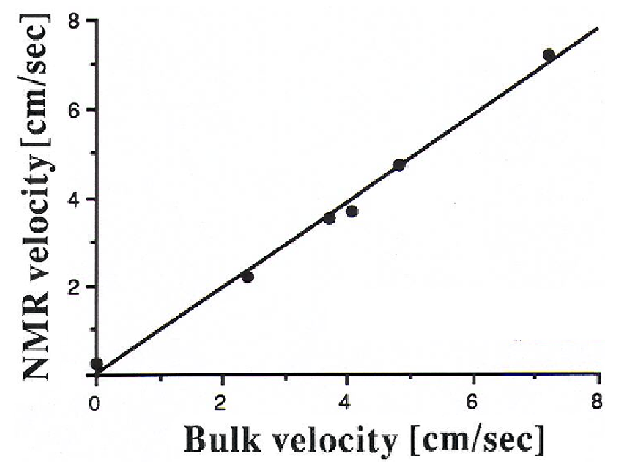

FIG. 5: Correlation between the average velocity NMR velocity measured using Half- Fourier Echo-Planar Imaging with the value of the bulk velocity determined from the known flow rate and the geometry of the tube.

crement can be observed as a function of the flow rate in this region. It is very unlikely that running water from a tap can reach a complete laminar regimen since may factors affect the behaviour of the water flow. These velocity fluctuations do not drastically affect the velocity measurements as shown in [11], however these small fluctuations can be measured with HF EPI. For $\operatorname{Re}>2500$ velocity fluctuation values grow rapidly with flow rate.

Our data is in good agreement with this well-known and established threshold of turbulence. It then seems that our flow MRI technique is able to detect velocity fluctuations which are
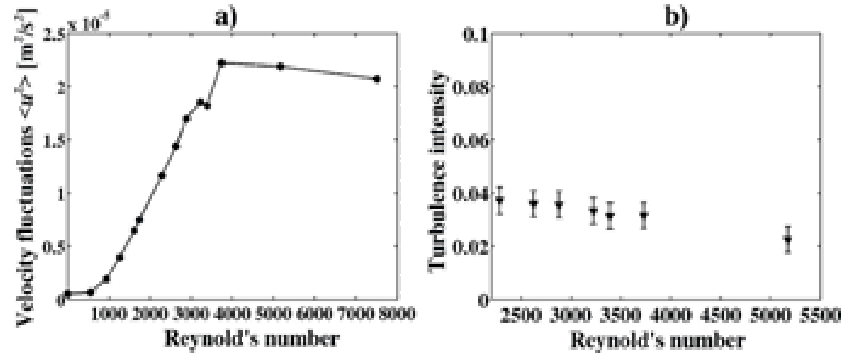

FIG. 6: a) Experimental velocity fluctuation $\left(\left\langle u^{2}\right\rangle\right)$ plotted against Reynolds number for pipe flow. a) Experimental turbulent intensity (TI $=\left\langle u^{2}\right\rangle^{1 / 2} / U$ plotted against Reynold's number: $U$ is the flow velocity.

not necessary caused by the turbulent behaviour of the flow. This is probably due to the better temporal resolution of HF EPI. Fig. 6b) plots the turbulent intensity, for the flow rates at which turbulent flows has been established $(\operatorname{Re}>2500)$. The turbulent intensity is approximately constant which is in good agreement with data reported in [11]. The method proposed here allows to directly obtain experimental measurements of the velocity fluctuations via a phase map formed with the two images determined with the flow encoding sequence and Eqs. (2) and (3). Despite the lower spatial resolution of a low field scanner, our data shows good agreement with the values reported in the literature.

It has been proved that flow encoded EPI together with a reduced data acquisition method can provide us with a real-time technique to measure laminar behaviour and velocity fluctuations for flow in pipes for Reynolds up to 80000. Guilfoyle and coworkers' approach can be easily modified to derive an expression to directly measure turbulent intensity and velocity fluctuations. In simple pipe flow, the onset of turbulence can then be accurately detected without disturbing the flow. This scheme reveals the potential to obtain relevant flow information for both laminar and turbulent behaviour of pipe flow.

\section{Acknowledgements}

I wish to express my gratitude to Sir Peter Mansfield for his supervision and advise during this work.
[1] D. N. Guilfoyle, P. Gibbs, R. J. Ordidge, and P. Mansfield, Magn. Reson. Med. 18, 1 (1991).

[2] K. Kose, J. Magn. Reson. 92, 631 (1991).

[3] J. C. Gatenby, J. C. Gore, J. Magn. Reson. 121, 193 (1996).

[4] A. J. Sederman, M. D. Mantle, C. Buckley, and L. F. Gladden, J. Magn. Reson. 166, 182 (2004).

[5] S. I. Han, P. T. Callaghan, J. Magn. Reson. 148, 349 (2001).

[6] Y. Q. Song, Ulrich M. Scheven, J. Magn. Reson. 172, 31 (2005).

[7] K. Kose, J. Phys. D: Appl. Phys. 23, 981 (1990).
[8] A. J. Sederman, M. D. Mantle, and L. F. Gladden, J. Magn. Reson. 161, 15 (2003)

[9] A. Rodriguez, B. Issa, R. Bowtell, and P. Mansfield, IV, Supp. 254, 100 (1996)

[10] A. M. Howseman, M. K. Stehling, B. Chapman, R. Coxon, R. Turner, R. J. Ordidge, M. G. Cawley, P. Glover, P. Mansfield, and R. E. Coupland, Br. J. Radiol. 61, 822 (1988).

[11] J. C. Gatenby, J. C. Gore, J. Magn. Reson. 110, 26 (1994) 